\title{
Effects of amount of excess solid, the type of stirring and sedimentation time on solubility of sodium phenytoin and lamotrigine
}

\author{
Shahrzad Moattar Mohammadi ${ }^{1,2}$, Ali Shayanfar ${ }^{3}$, Shahram Emami $^{3}$, Abolghasem \\ Jouyban $^{4}$ \\ ${ }^{1}$ Biotechnolgy Research Center, Tabriz University of Medical Sciences, Tabriz, Iran \\ ${ }^{2}$ Student Research Committee and Faculty of Pharmacy, Tabriz University of Medical Sciences, Tabriz, Iran \\ ${ }^{3}$ Drug Applied Research Center and Faculty of Pharmacy, Tabriz University of Medical Sciences, Tabriz, Iran \\ ${ }^{4}$ Pharmaceutical Analysis Research Center and Faculty of Pharmacy, Tabriz University of Medical Science, Tabriz, Iran
}

*Corresponding Author: E-mail: shayanfara@tbzmed.ac.ir; Tel.: +984133341315; Fax: +1-111-111-112

Received: September 27, 2018; Revised: October 22, 2018; Published: November 03, 2018

\begin{abstract}
Solubility is the maximum quantity of a drug dissolved in a given volume of solvent at a specific temperature. Several factors affect equilibrium solubility. Therefore, different solubility data have been reported for a solute in a certain solvent and temperature in the literature. These variations in solubility are one of the possible reasons for unsuccessful attempts of medicinal chemists for developing models as well as deviation of experimental works for solubility prediction in aqueous, non-aqueous and solvent mixtures. The present research aim is to investigate the effect of the amount of excess solid and the type of stirring on the solubility of drugs. The solubility of two antiepileptic drugs, namely sodium phenytoin and lamotrigine was determined in water, ethanol, and $\mathrm{HCl} 0.1 \mathrm{M}$ at $37^{\circ} \mathrm{C}$. Different excess amounts of drugs were added to the constant volume of solvent. Additionally, different stirring methods such as magnetic stirrer and shake-flask and sedimentation time were investigated on the solubility values. Saturation solubility of drugs after dilution with water was measured using a spectrophotometer, and the concentration was calculated according to the calibration curve. Amount of excess solid, especially when the drug is in ionized form, and sedimentation time after $24 \mathrm{~h}$ have a significant effect on solubility values.
\end{abstract}

\section{Keywords}

Antiepileptic drugs; equilibrium solubility; determination

\section{Introduction}

Solubility of drugs is an important aspect from the earliest stages of drug discovery to the latest stage of the drug formulation [1,2]. It is defined as the maximum quantity of a drug dissolved in a given volume of a solvent at a certain temperature [3]. Solubility data for a solute, especially for pharmaceuticals, is a significant physicochemical property in crystallization, extraction of an analyte from different matrices, evaluation of oral bioavailability and preparation of liquid and semi-solid dosage forms. Furthermore, solubility in water is needed to make a solution of the drug or drug-like molecule to be tested for its pharmacological/toxicological activities. Despite simple methods developed for experimental 
determination of the solubility of drugs [4], various parameters can affect accuracy of the obtained data [5]. Several different sets of solubility data have been reported for a drug at a certain solvent and temperature in the literature [6-8].

These solubility variations are one of the possible reasons for unsuccessful attempts of medicinal chemist in developing models for solubility prediction in water, non-aqueous solvents and solvent mixtures with deviation in agreement with experimental works. For example, the best model for aqueous solubility prediction has a prediction error more than $100 \%$ [9], and estimation error for solubility in the solvent mixture is higher than $25 \%$ for pharmaceuticals [6]. There are various methods for solubility determination of pharmaceuticals $[10,11]$. The shake-flask method is a common method for solubility determination which an excess amount of drug is added to a certain solvent and after appropriate shaking at a constant temperature to reach the equilibrium condition, and the saturated concentration is determined by a valid analytical method [11]. In some cases, variation of solubility data originates from the nature of studied compounds, such as polymorphism and formations of drug aggregates, micelles, drug-buffer complexes, stability, solute and solvent impurities; they can affect the solubility value. Another problem in solubility determination is invalidity of the applied method for quantification of drug concentration. Other sources of variations, which can affect solubility values related to the solubility determination process such as time of equilibrium, incomplete dissolution over the equilibration time and inappropriate phase separation solution handling and adsorption to untreated surfaces, amount of residual solid, speed and stirring or shaking patterns of the suspension $[5,7,12]$. Although, various sets of solubility data for chemical and pharmaceutical materials in water and organic solvents are annually collected by different research groups and reported in the literature, there are few investigations into the effect of different parameters on solubility determination of drugs. Baka et al. [8] investigated the effect of various parameters such as amount of solid excess, stirring time, temperature, and sedimentation time on solubility of hydrochlorothiazide as a model drug. Some reports have been investigated the effect of excess solid on solubility of drugs [13,14]; and the reported results are discussed in review articles [11,15].

Lamotrigine is a basic drug $\left(\mathrm{p} K_{\mathrm{a}}=5.7\right)$ [16] existing in unionized form in water, while it is in ionized form in $0.1 \mathrm{M} \mathrm{HCl}$. Sodium phenytoin is a salt form of phenytoin (an acidic compound with $\mathrm{p} K_{\mathrm{a}}=8.3$ [16]) being partially ionized form in water. In addition, it changes to the parent drug (un-ionized form) in acidic medium. In this study, the effects of the amount of solid excess, shaking patterns of solution and sedimentation time were investigated on solubility of two antiepileptic drugs, namely sodium phenytoin and lamotrigine in water, ethanol and acidic medium ( $\mathrm{HCl} 0.1 \mathrm{M})$.

\section{Experimental}

\section{Samples and chemicals}

Sodium phenytoin (99\%) and lamotrigine (99\%) were purchased from Alhavi Pharmaceutical Company (Tehran, Iran). Ethanol (99\%) was provided from Scharlau (Barcelona, Spain) and $\mathrm{HCl}$ (37\%) was supplied from Merck (Darmstadt, Germany), and double distilled water was used as received in the lab.

\section{Apparatus}

A shaker incubator (Heidolph, Germany) or a magnetic stirrer (Heidolph MR Hei-Tec, Germany) was used to mix two phases and maintain the temperature of samples at $37 \pm 0.1{ }^{\circ} \mathrm{C}$ during the solubility measurements. Samples were filtered using a hydrophilic membrane (450 nm pore size). The concentration of the saturated solution was measured using a UV-Vis spectrophotometer (Shimadzu, 
Japan). The $\mathrm{pH}$ of the solutions was measured by a $\mathrm{pH}$ meter (Metrohm, Switzerland).

Measurement of solubility and investigation of the effect of excess amount of solute and type of stirring on solubility

Excess amount of each drug was added to a small volume (e.g. $5 \mathrm{~mL}$ ) of the studied solvent in an Erlenmeyer flask. The amount of excess was added according to the preliminary experiments or reported solubility of drugs in the studied solvent from the literature (solubility of phenytoin in water and ethanol are $86 \mathrm{mg} / \mathrm{ml}$ and $19.9 \mathrm{mg} / \mathrm{mL}$ at $35{ }^{\circ} \mathrm{C}$ [17], respectively and lamotrigine solubility in water and $\mathrm{HCl} 0.1 \mathrm{M}$ are $0.19 \mathrm{mg} / \mathrm{ml}$ [18] and $2.22 \mathrm{mg} / \mathrm{mL}$ [19] at $25{ }^{\circ} \mathrm{C}$, respectively). Therefore, in this study, the volume of solvent was considered $5 \mathrm{~mL}$ in an Erlenmeyer flask, being similar to the routine experiment of solubility determination in the pharmaceutical and chemical laboratories. For highly soluble compounds, e.g. sodium phenytoin in water $10 \%, 25 \%, 100 \%, 150 \%$ and $200 \%$ of saturated solubility of the drug was applied as an excess solid amount. While for low solubility values, e.g. lamotrigine in water, the minimum amount of drug could be weighted by a digital balance (the minimum amount of weighting $10-30 \mathrm{mg}$ ), was selected as the lowest concentration of suspension for solubility determination and other solutions up to 5 -folds of this amount were prepared to simulate a routine solubility determination experiment. Then, the samples were placed in an incubator equipped with a temperature-controlling system at $37^{\circ} \mathrm{C}$ in $150 \mathrm{rpm}$ for 48 hours. Afterward, the saturated solutions of the drugs were filtered using hydrophilic filters $(0.45 \mu \mathrm{m})$. In addition, magnetic stirrer and shake-flask approaches, separately, were used to saturate sodium phenytoin and lamotrigine in water at $37^{\circ} \mathrm{C}$ and $150 \mathrm{rpm}$ for 48 hours. Furthermore, the concentration of samples was measured after 24-hour sedimentation. The concentration of samples was measured by a UV spectrophotometer. Each datum was obtained from at least three replicate independent measurements, and the maximum relative standard deviation (RSD) of each experiment was $10 \%$.

\section{Results}

The effect of the excess solid amount on solubility of sodium phenytoin in water, ethanol and $\mathrm{HCl}(0.1 \mathrm{M})$

Figure 1 shows the solubility of sodium phenytoin in water at $37^{\circ} \mathrm{C}$. The experiment was investigated in various excess amounts of phenytoin sodium. A linear relationship exists between excess solid amount of drug and solubility. The percentage change between the maximum and minimum values of solubility is $116 \%$; however, the excess amount has a low effect on solubility of phenytoin in $\mathrm{HCl} 0.1 \mathrm{M}$ (Figure 2) in comparison to solubility in water (percentage change was less than $30 \%$ ). Moreover, to confirm the effect of excess amount in ionized form, phenytoin solubility was performed in ethanol (dielectric constant of ethanol is lower than water and the ionization in ethanol is less than water) and the results were illustrated in Figure 3. A similar pattern to $\mathrm{HCl}$ (percentage difference was $21 \%$ ) was obtained for solubility of sodium phenytoin in ethanol.

The effect of the excess solid amount on solubility of lamotrigine in water, ethanol and $\mathrm{HCl}(0.1 \mathrm{M})$

Figure 4 illustrates solubility of lamotrigine in water at $37^{\circ} \mathrm{C}$ in different amounts of excess solid, and the obtained data revealed that excess amount had no significant effect on solubility value. However, it has a considerable effect on solubility value in $\mathrm{HCl} 0.1 \mathrm{M}$ (Figure 5, the percentage difference between the maximum and minimum amount of solubility value was higher than $40 \%$ ), which lamotrigine is in an ionized form similar to the data obtained for phenytoin sodium. 
The effect of type of stirring and sedimentation time on solubility of sodium phenytoin and lamotrigine in water

Magnetic stirrer and shake-flask approaches were used to saturate sodium phenytoin and lamotrigine in water at $37^{\circ} \mathrm{C}$. Figure 6 illustrates the effect of type of stirring on the obtained solubility value, and shown, the type of stirring for solubility determination has no significant effect on the saturated concentration (solubility) of phenytoin sodium. Moreover, sedimentation time after 24-hour (Figures 7 and 8) was investigated on the solubility values, and a significant change was observed in solubility of sodium phenytoin in both stirring instruments. Similar results were observed for solubility determination of lamotrigine in water (Figure 9). The data indicate that sedimentation time seems to have a significant effect on solubility value, particularly for stirring of the solution with magnet stirrer.

\section{Discussion}

\section{The effect of excess amount on solubility of sodium phenytoin and lamotrigine}

The study results show that excess solid amount of the studied drugs has a considerable effect on solubility, especially in ionized form i.e. sodium phenytoin in water and lamotrigine in $\mathrm{HCl}(0.1 \mathrm{M})$. One of the reasons for change in solubility in the presence of different values of excess solid is change in $\mathrm{pH}$ of saturated solution [20]. In this study, solubility was determined in aqueous solution at own $\mathrm{pH}$. However, $\mathrm{pH}$ determination of final solutions (various excess amounts) showed no substantial change (pH: 11.6 \pm 0.05 ). A differential scanning calorimetry (DSC) study of the remaining solid of lamotrigine confirms no possible change in crystalline phase due to a sharp peak corresponding to lamotrigine melting at $220^{\circ} \mathrm{C}$, being in agreement with the literature [19]. However, DSC thermogram sodium phenytoin in water exhibited some new peaks resultant to phase transformation of drug to the hexahydrate form similar to the reported study by Rubino [21], and it is not possible to judge about the polymorphic change or dimer formation whenever the amount of excess solid is changed.

Kawakami et al. [13] have reported that solubility of indomethacin increased with an increase in the solid amount in ionizable form ( $\mathrm{pH}=6.5-7)$ against indomethacin solubility in non-ionizable form $(\mathrm{pH}=5)$. They discussed the anomalous behavior in dissolution and crystallization rates at near equilibrium conditions as the possible mechanism. However, one of the potential reasons based on Avdeef's comment on that study is the possible partitioning (adsorption) of the charged form of indomethacin onto the excess solid present in the suspensions, and some ionizable compounds may be surface-active. However, further mechanisms such as dimer formation are possible, and the results are not interpretable without analysis of the solid form [15].

Nevertheless, a similar research study about solubility measurement of hydrochlorothiazide reported no significant difference for solubility values in water when different excess amounts of the drug were used [8]. Hydrochlorothiazide as an acidic compound converts to non-ionized form in saturated aqueous solution. Another study by Mosharraf et al. [22] reported that solubility of chemical compounds (in salt form) was affected by the amount of excess solid. Increasing the amount of excess solid of barium sulphate or calcium carbonate can affect solubility value due to the disordering particle surface and existence of a peripheral disordered layer.

According to the obtained study, solubility of chemical compounds can change by the amount of excess solid, and the mutual issue between the reported studies is ionization and salt form of solute. Change in the diffusion layer and disordering particle surface due to adsorption of the charged form of the drug and 
surface activity of solute in ionized form could be an acceptable interpretation for increasing solubility values in the higher amount of excess solids. Similar phenomena have been proposed for increase in saturation solubility of nanocrystals (size of particle less than $1000 \mathrm{~nm}$ ) by the change in layer of unstirred layer surrounding the drug particle (diffusion layer) [23]. Moreover, when drug salts are added to solution, their solubility is expected to vary according to the amount of solid added, since the solubility product may not be satisfied below a certain amount of added salt. The partial conversion back of sodium phenytoin to free acid form during solubility testing reported by Chaing and Wang [24] could be reason for variability in the solubility values.

\section{The effect of type of stirring and sedimentation time on solubility of lamotrigine and phenytoin sodium}

Type of stirring is a critical parameter in evaluating dissolution of pharmaceuticals [24]. However, it has been not yet considered as an effective factor in solubility determination of solute. Magnetic stirrer and shake-flask are common approaches in the literature to attain the solution to equilibrium. Type of stirring could be considered as a possible reason for the variation of solubility values because of supersaturation phenomenon. It is a state of a solution that contains more of the dissolved material than could be dissolved in the solvent [7]. The results in Figures 6-9 show that type of stirring has a slight effect on obtained solubility for lamotrigine and phenytoin sodium. However, sedimentation time after 24-hour, in both types of stirring, has a considerable effect on solubility values. In this regard, Baka et al. [8] conducted the only study about the effect of sedimentation time for solubility of hydrochlorothiazide; similar to the results of this study, sedimentation time had a considerable effect on the aqueous solubility of drug.

The results of this study indicate that although solubility determination of drugs seems a simple experiment, the numerical values of solubility of a compound are affected by different factors. Amount of excess solid and sedimentation time could have a significant effect on solubility values.

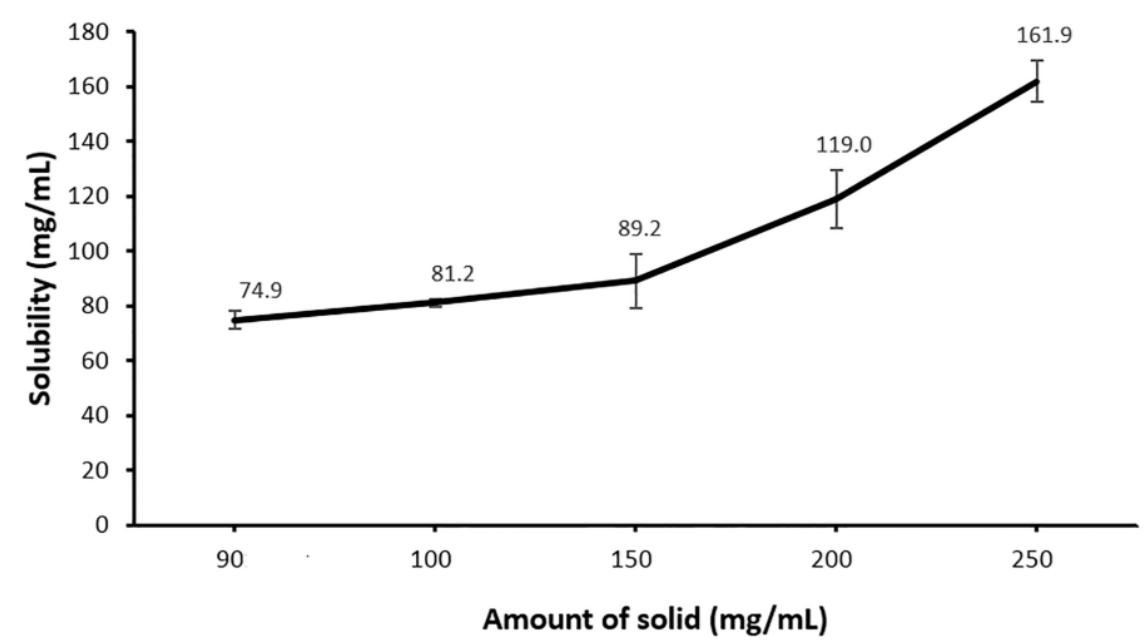

Figure 1. The effect of excess solid amount on solubility of sodium phenytoin in water at $37{ }^{\circ} \mathrm{C}$ (added weight of drug associated with each of the points in $5 \mathrm{~mL}$ of solution is $450,500,750,1000,1250 \mathrm{mg}$, respectively). 


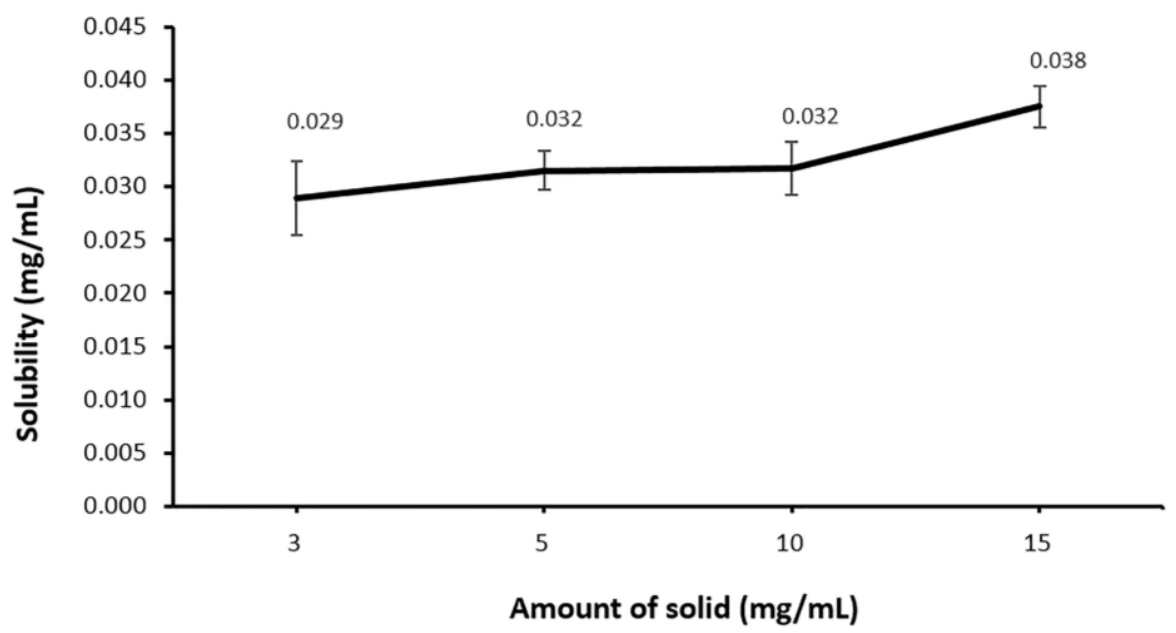

Figure 2. The effect of the excess solid amount on solubility of sodium phenytoin in $\mathrm{HCl} 0.1 \mathrm{M}$ at $37^{\circ} \mathrm{C}$. (Added weight of drug associated with each of the points in $5 \mathrm{~mL}$ of solution is 15, 25, 50 and $75 \mathrm{mg}$, respectively).

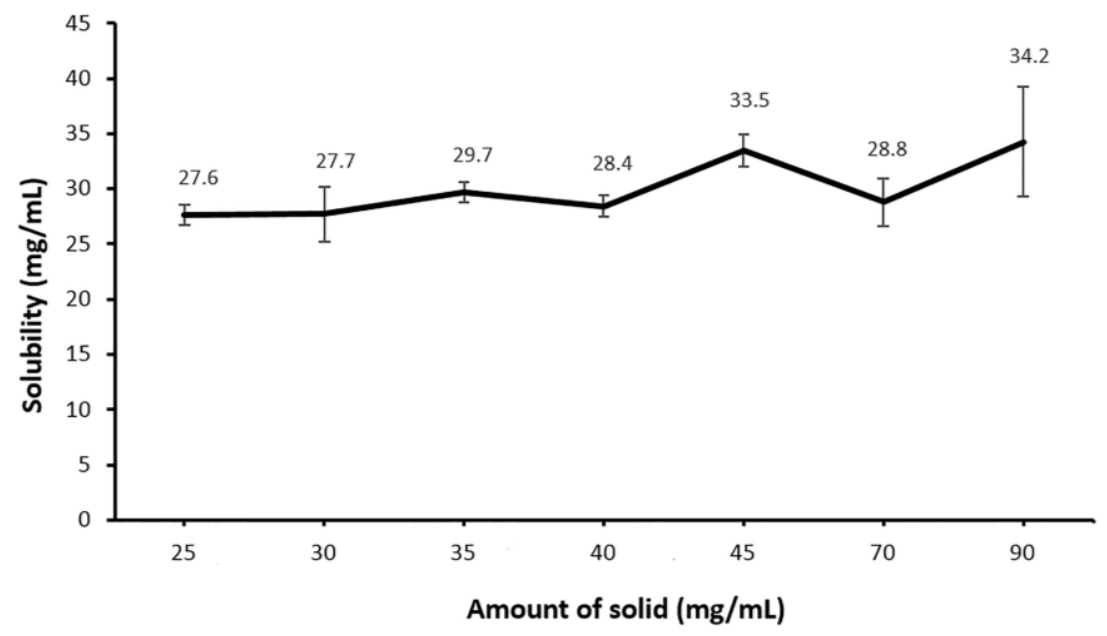

Figure 3. The effect of the excess solid amount on solubility of sodium phenytoin in ethanol at $37{ }^{\circ} \mathrm{C}$. (Added weight of drug associated with each of the points in $5 \mathrm{~mL}$ of solution is $125,150,175,200,225,350$ and 450 $\mathrm{mg}$, respectively).

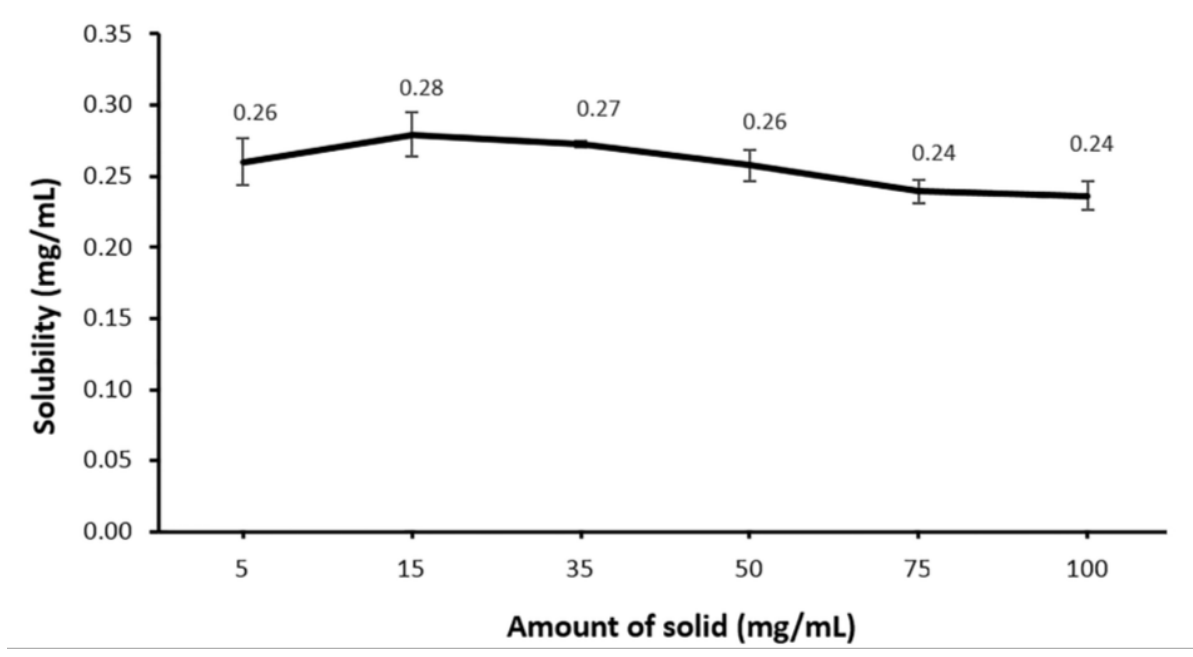

Figure 4. The effect of the excess solid amount on solubility of lamotrigine in water at $37^{\circ} \mathrm{C}$. (Added weight of drug associated with each of the points in $5 \mathrm{~mL}$ of solution is $25,75,175,250,375,500 \mathrm{mg}$, respectively). 


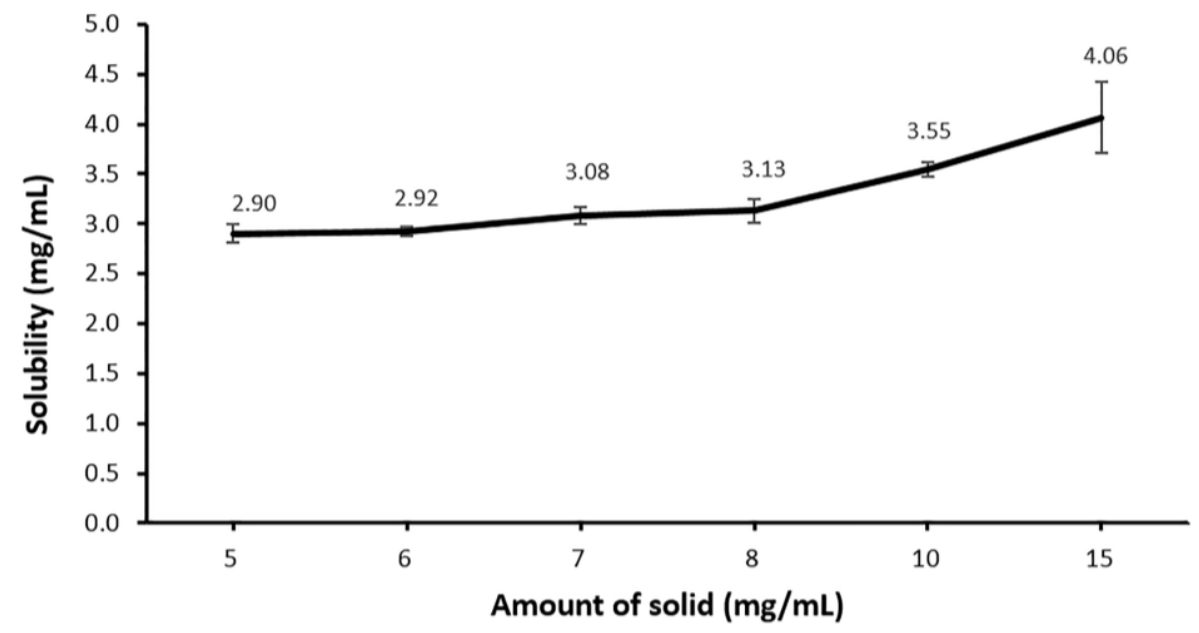

Figure 5. The effect of the excess solid amount on solubility of lamotrigine in $\mathrm{HCl}(0.1 \mathrm{M})$ at $37^{\circ} \mathrm{C}$. (Added weight of drug associated with each of the points in $5 \mathrm{~mL}$ of solution is $25,30,35,40,50$ and $75 \mathrm{mg}$, respectively).

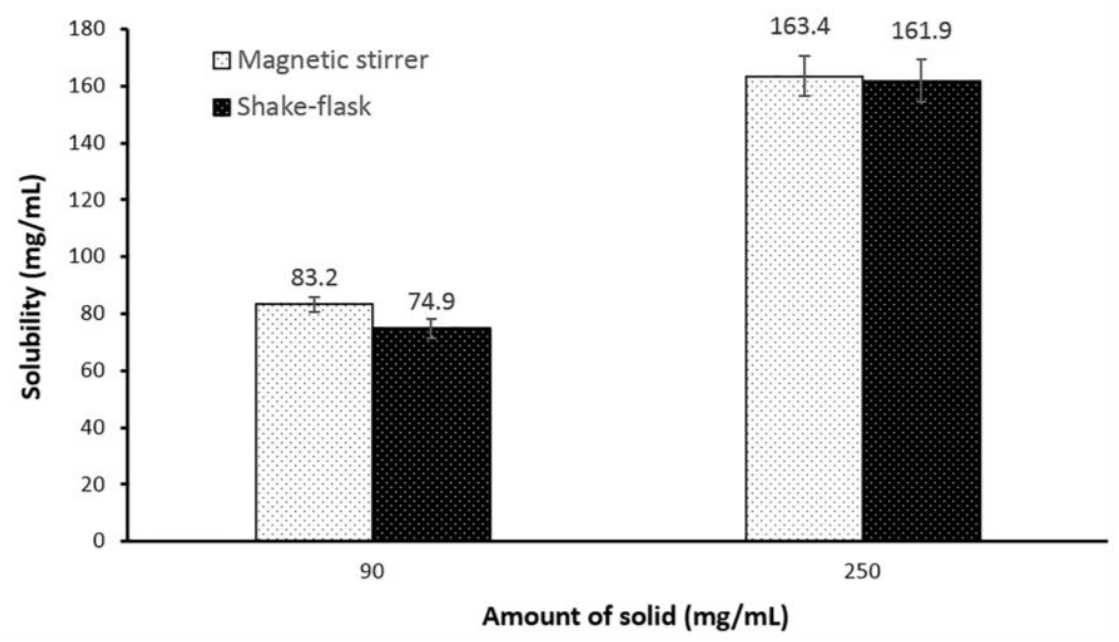

Figure 6. The effect of type of stirring on sodium phenytoin solubility in water at $37{ }^{\circ} \mathrm{C}$. (Added weight of drug associated with each of the points in $5 \mathrm{~mL}$ of solution is $450 \mathrm{mg}$ and $1250 \mathrm{mg}$ ).

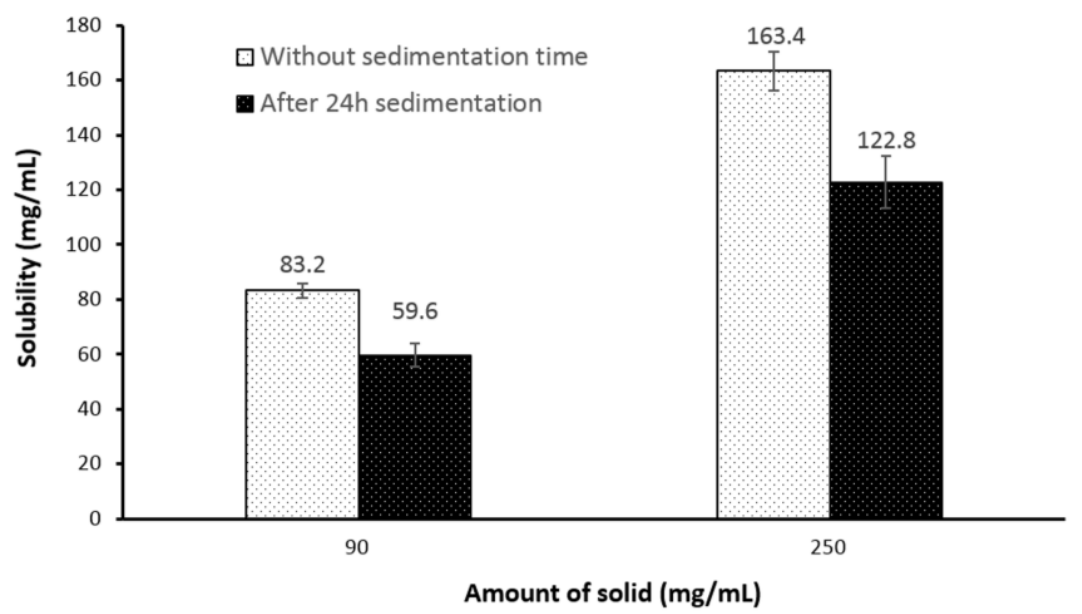

Figure 7. The effect of sedimentation on sodium phenytoin solubility in water at $37{ }^{\circ} \mathrm{C}$ (the suspensions were stirred with magnetic-stirrer). (Added weight of drug associated with each of the points in $5 \mathrm{~mL}$ of solution is $450 \mathrm{mg}$ and $1250 \mathrm{mg}$, respectively). 


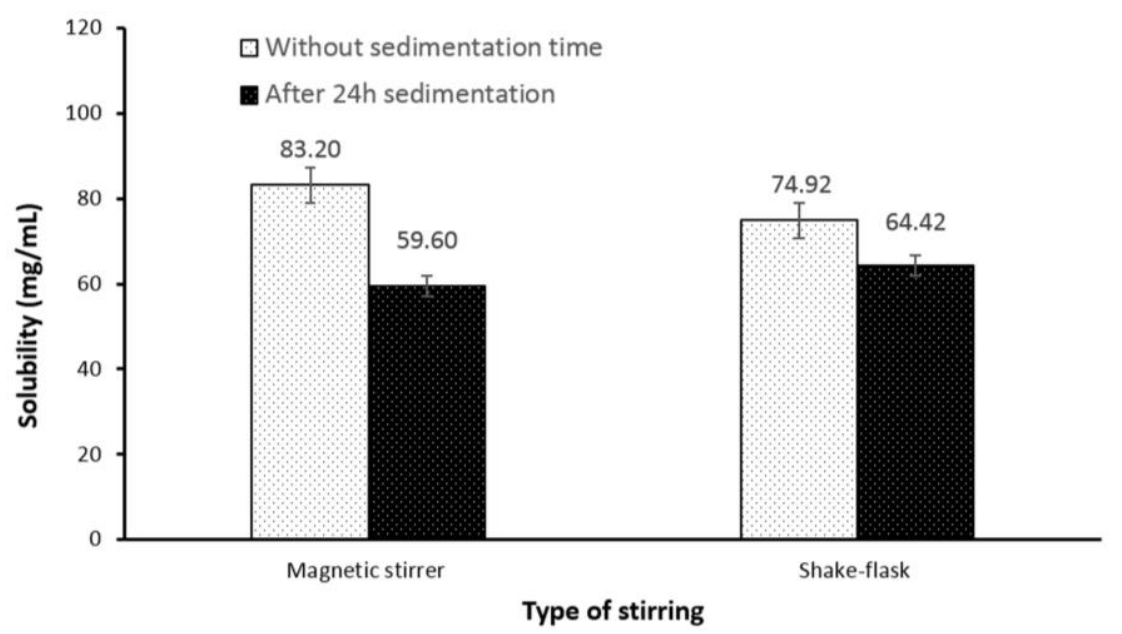

Figure 8. The effect of sedimentation on sodium phenytoin solubility in water at $37{ }^{\circ} \mathrm{C}$ (the amount of excess solid was $450 \mathrm{mg} / 5 \mathrm{~mL}$ ).

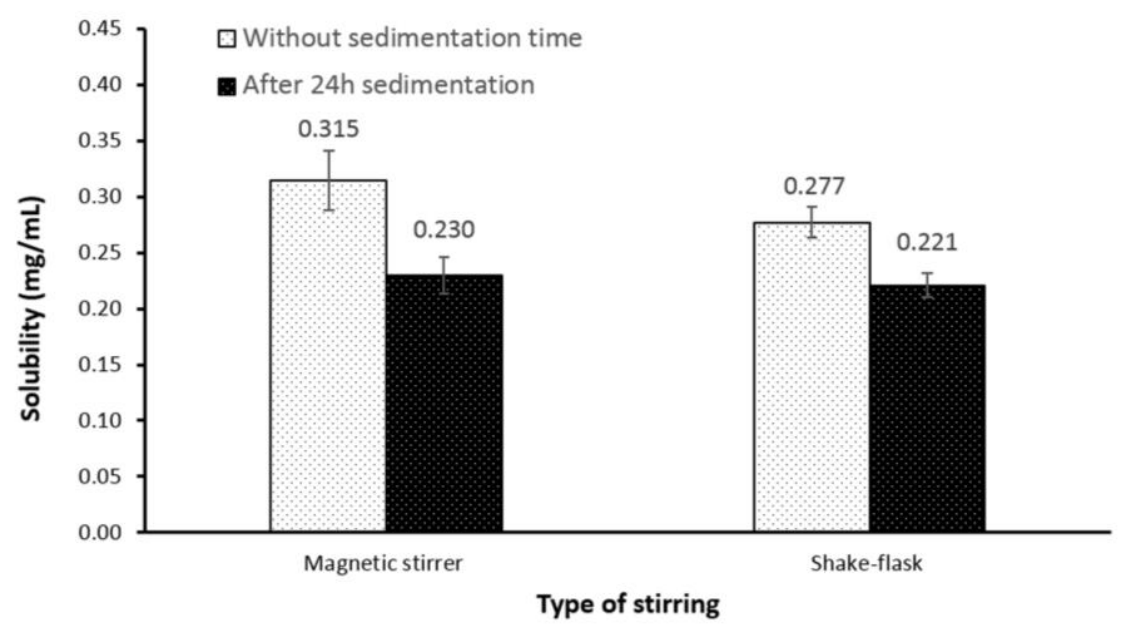

Figure 9. The effect of sedimentation on lamotrigine solubility in water at $37^{\circ} \mathrm{C}$ (the amount of excess solid was $175 \mathrm{mg} / 5 \mathrm{~mL})$.

\section{Conclusion}

Solubility is a classic and common physicochemical property of pharmaceuticals; however, its values are affected by various parameters. The findings of this study indicated that the amount of excess solid, especially in ionized form and sedimentation time after appropriate stirring, had significant effects on the obtained solubility of sodium phenytoin and lamotrigine. The findings show that these factors are possible reasons for variation in the reported solubility data in the literature and relatively unsuccessful attempts to develop solubility prediction models. It seems that the proper reporting of experimental details is necessary in solubility studies.

\section{Acknowledgements}

This article is a part of the results of S.M's Pharm.D thesis No. 85 registered at Faculty of Pharmacy, Tabriz University of Medical Sciences, Tabriz, Iran. A.S. thanks the Ministry of Health and Medical Education (grant for young assistant professors), Tehran, Iran, for financial support. 


\section{References}

[1] L. Di, P.V. Fish, T. Mano. Bridging solubility between drug discovery and development. Drug Discov.Today 17 (2012) 486-495.

[2] F. Martínez, A. Jouyban, W.E. Acree. Pharmaceuticals solubility is still nowadays widely studied everywhere. Pharm. Sci. 23 (2017) 1-2.

[3] A.T. Florence, D. Attwood, Physicochemical Principles of Pharmacy, Pharmaceutical Press, London, 2006.

[4] A. Jouyban, M.A.A. Fakhree, Experimental and Computational Methods Pertaining to Drug Solubility, in: W.E. Acree, Jr. (Ed.) Toxicity and Drug Testing, InTech Publisher: New York, 2012.

[5] J. Alsenz, M. Kansy. High throughput solubility measurement in drug discovery and development. Adv. Drug Deliv. Rev. 59 (2007) 546-567.

[6] A. Jouyban. Review of the cosolvency models for predicting solubility of drugs in water-cosolvent mixtures. J. Pharm. Pharm. Sci. 11 (2008) 32-58.

[7] A. Avdeef, E. Fuguet, A. Llinàs, C. Ràfols, E. Bosch, G. Völgyi, T. Verbić, E. Boldyreva, K. Takács-Novák. Equilibrium solubility measurement of ionizable drugs-consensus recommendations for improving data quality. ADMET \& DMPK 4 (2016) 117-178.

[8] E. Baka, J.E.A. Comer, K. Takács-Novák. Study of equilibrium solubility measurement by saturation shake-flask method using hydrochlorothiazide as model compound. J. Pharm. Biomed. Anal. 46 (2008) 335-341.

[9] A. Shayanfar, M.A.A. Fakhree, A. Jouyban. A simple QSPR model to predict aqueous solubility of drugs. J. Drug Deliv. Sci. Technol. 20 (2010) 467-476.

[10] A. Ghanbari, Y. Sarbaz, V. Jouyban-Gharamaleki, K. Jouyban-Gharamaleki, J. Soleymani, A. Jouyban. An improved automated setup for solubility determination of drugs. Pharm. Sci. 22 (2016) 210-214.

[11] S.B. Murdande, M.J. Pikal, R.M. Shanker, R.H. Bogner. Aqueous solubility of crystalline and amorphous drugs: Challenges in measurement. Pharm. Dev. Technol. 16 (2011) 187-200.

[12] A. Avdeef. Suggested improvements for measurement of equilibrium solubility-pH of ionizable drugs. ADMET \& DMPK 3 (2015) 84-109.

[13] K. Kawakami, K. Miyoshi, Y. Ida. Impact of the amount of excess solids on apparent solubility. Pharm. Res. 22 (2005) 1537-1543.

[14] Z. Wang, L.S. Burrell, W.J. Lambert. Solubility of E2050 at various pH: A case in which apparent solubility is affected by the amount of excess solid. J. Pharm. Sci. 91 (2002) 1445-1455.

[15] A. Avdeef. Solubility of sparingly-soluble ionizable drugs. Adv. Drug Deliv. Rev. 59 (2007) 568-590.

[16] A.C. Moffat, M.D. Osselton, B. Widdop, J. Watts, Clarke's analysis of drugs and poisons, Pharmaceutical Press, London, 2011.

[17] A. Mabhoot, A. Jouyban. Solubility of sodium phenytoin in ethanol + water mixtures at various temperatures. Chem. Eng. Technol. 203 (2016) 1009-1012.

[18] A. Shayanfar, M.A.A. Fakhree, W.E. Acree Jr, A. Jouyban. Solubility of lamotrigine, diazepam, and clonazepam in ethanol + water mixtures at 298.15 K. J. Chem. Eng. Data 54 (2009) 1107-1109.

[19] K. Beattie, G. Phadke, J. Novakovic, Chapter 6 - Lamotrigine, In: H.G. Brittain (Ed.) Profiles of Drug Substances, Excipients and Related Methodology, Academic Press, 2012, pp. 245-285.

[20] A. Avdeef, Absorption and drug development: solubility, permeability, and charge state, John Wiley \& Sons, Hoboken, New Jersey, 2012.

[21] J.T. Rubino. Solubilities and solid state properties of the sodium salts of drugs. J. Pharm. Sci. 78 (1989) 485-489.

[22] M. Mosharraf, T. Sebhatu, C. Nyström. The effects of disordered structure on the solubility and dissolution rates of some hydrophilic, sparingly soluble drugs. Int. J. Pharm. 177 (1999) 29-51. 
[23] F. Nielloud, Pharmaceutical emulsions and suspensions: revised and expanded, CRC Press, Boca Raton, 2000.

[24] J. Bevernage, J. Brouwers, M.E. Brewster, P. Augustijns. Evaluation of gastrointestinal drug supersaturation and precipitation: Strategies and issues. Int. J. Pharm. 453 (2013) 25-35.

(C)2018 by the authors; licensee IAPC, Zagreb, Croatia. This article is an open-access article distributed under the terms and conditions of the Creative Commons Attribution license (http://creativecommons.org/licenses/by/3.0/) (cc)) $\mathrm{EY}$ 